\title{
Observations sur l'effet du système des manufactures (1817) et Enquête ouvrière (1880)
}

\section{Robert Owen et Karl Marx}

\section{OpenEdition}

\section{Journals}

Édition électronique

URL : http://journals.openedition.org/grm/2388

DOI : $10.4000 / \mathrm{grm} .2388$

ISSN : $1775-3902$

\section{Éditeur}

Groupe de Recherches Matérialistes

\section{Référence électronique}

Robert Owen et Karl Marx, "Observations sur l'effet du système des manufactures (1817) et Enquête ouvrière (1880) », Cahiers du GRM [En ligne], 16 | 2020, mis en ligne le 07 juillet 2020, consulté le 27 décembre 2020. URL : http://journals.openedition.org/grm/2388; DOI : https://doi.org/10.4000/grm. 2388

Ce document a été généré automatiquement le 27 décembre 2020.

(c) GRM - Association 


\title{
Observations sur l'effet du système des manufactures (1817) et Enquête ouvrière (1880)
}

\author{
Robert Owen et Karl Marx
}

\section{Robert Owen : «Observations sur l'effet du système des manufactures avec des suggestions pour améliorer ses aspects qui portent le plus d'attente à la santé et à la morale, dédiées avec le plus grand respect au corps législatif britannique ${ }^{7}$}

1 Ceux qui se consacrèrent il y a trente ou quarante ans au commerce et aux manufactures dans ce pays ne représentaient qu'une partie très négligeable du savoir, de la richesse, du pouvoir ou de la population au sein de l'Empire.

2 Avant cette période-là, l'Empire britannique reposait essentiellement sur l'agriculture. Mais, depuis cette époque-là jusqu'à aujourd'hui, les échanges intérieurs et ceux avec l'étranger se sont amplifiés d'une manière tellement rapide et extraordinaire qu'ils ont donné au commerce une importance qu'il n'avait jamais obtenue auparavant dans un pays doté d'un tel pouvoir et d'une telle influence sur le plan politique ${ }^{2}$.

3 De cette transformation, les principaux responsables ont été les inventions mécaniques qu'ont introduit le commerce du coton dans ce pays et la culture du cotonnier en Amérique. Les demandes que ce commerce a créées concernant les différentes matières requises pour impulser ses opérations multipliées a produit une demande extraordinaire pour presque toutes les manufactures déjà existantes et, évidemment, pour le travail humain ${ }_{8 \mathrm{~B}}^{28}$ human labour ${ }_{80}^{29}$.

4 Nombreux tissus pleins d'imagination et d'utilité produits par la manufacture à partir du coton devinrent rapidement des objets de désire en Europe et en Amérique ; et l'extension correspondante du commerce extérieur anglais avec l'étranger fut telle 
qu'elle étonna et frappa de stupeur les hommes d'État les plus éclairés tant en Angleterre qu'à l'étranger.

5 L'effet immédiat de ce phénomène manufacturier fut l'augmentation rapide de la richesse, de l'industriosité, de la population et du poids politique de l'Empire britannique, grâce à quoi ce dernier fut capable de se battre, pendant vingt-cinq ans, contre la puissance la plus formidable militairement et la plus immorale jamais vue dans le monde.

6 Ces résultats importants néanmoins, malgré leur réelle grandeur, n'ont pas pu être obtenus sans être accompagnés de maux d'une telle dimension qu'ils poussent à se demander s'ils ne s'avèrent pas prépondérants par rapport aux biens.

7 Jusqu'ici, les législateurs ont semblé ne considérer les manufactures que d'un seul point de vue, c'est-à-dire en tant que source de la richesse nationale. Les autres conséquences d'envergure qui surgissent de l'extension des manufactures, lorsque ces dernières sont laissées à leur marche naturelle, n'ont jamais attiré l'attention d'aucun corps législateur. Pourtant, les effets politiques et moraux auxquels nous faisons allusion, méritent tout à fait d'occuper les facultés les plus développées des hommes d'État les plus grands et les plus sages.

8 La diffusion générale des manufactures sur toute l'étendue du pays engendre un nouveau caractère chez ses habitants; et, étant donné que ce caractère repose sur un principe tout à fait défavorable au bonheur individuel et général, il finira par produire les maux les plus déplorables et persistants, du moins si sa tendance ne sera pas contrecarrée par une intervention et une direction législatives.

9 Le système des manufactures a déjà tellement étendu son emprise sur l'Empire britannique qu'un changement essentiel s'en est suivi dans le caractère général de la masse du peuple. Cette altération est toujours en train de s'accomplir rapidement; et bientôt, la simplicité comparativement heureuse du paysan et du travailleur agricole sera complètement perdue parmi nous. Déjà maintenant, elle est difficile à trouver sans qu'elle soit mélangée à ces coutumes qu'engendrent le commerce et la manufacture.

10 L'acquisition de la richesse, e le désire qu'elle engendre naturellement d'une augmentation continue, ont introduit un goût pour des formes nuisibles de luxe chez des vastes populations d'individus qui n'y auraient jamais songé auparavant, et ont produit une disposition qui stimule fortement ceux qui en sont affectés à sacrifier les sentiments les meilleurs de la nature humaine devant cet amour de l'accumulation. Afin de réussir cette démarche, l'activité industrieuse des classes inférieures, du travail desquelles cette richesse est tirée maintenant, a été amenée par des nouveaux acteurs menant une compétition féroce contre les entreprises établies depuis plus longtemps, à devenir une véritable oppression, en réduisant ces classes, à travers des changements successifs et au fur et à mesure que l'esprit de concurrence augmentait et que la facilité d'acquérir des richesses diminuait, à une condition plus misérable que tout ce qui peut être imaginé par ceux qui n'ont pas observé ces changements au cours de leur réalisation progressive. Ainsi, ces classes sont maintenant dans un état infiniment plus dégradé et misérable que celui dans lequel ils étaient avant l'introduction de ces manufactures, du succès desquelles dépend maintenant leur survie simple et nue.

11 Afin de substanter l'augmentation de population qu'a produite cette demande accrue de travail, il devient nécessaire maintenant de conserver le volume de notre commerce 
avec l'étranger, sous peine de le transformer, dans les circonstances données concernant la population, en un mal sérieux et inquiétant.

Il est hautement vraisemblable, toutefois, que le commerce d'exportation de ce pays ait atteint sa limite supérieure, et que, à cause de la concurrence d'autres États possédant des avantages locaux identiques ou supérieurs, il commence maintenant à s'affaiblir.

L'effet immédiat du Corn-bill consistera à accélérer ce déclin et à détruire précocement ce commerce-là. De ce point de vue, il est profondément regrettable qu'il soit devenu une loi; et je suis persuadé que ses partisans vont bientôt découvrir la nécessité absolue de l'abroger afin d'empêcher la misère qui devra s'en suivre pour la grande masse du peuple.

14 Les habitants de chaque nation sont élevés et modelés par les circonstances générales qui prévalent en elle, et le caractère des classes inférieures en Angleterre est actuellement modelé principalement par les circonstances qui se dégagent du commerce et de la manufacture; et le principe qui gouverne le commerce et la manufacture est le gain monétaire immédiat, devant lequel tous les autres avantages s'inclinent à l'échelle la plus vaste.

Tout le monde est diligemment entrainé pour acheter bon marché et pour vendre cher ; et, pour réussir cet art, chaque partie doit apprendre à acquérir un pouvoir considérable de tromper son prochain. Ainsi, un esprit est engendré chez chaque catégorie de commerçants susceptible de détruire cette sincérité honnête et ouverte sans laquelle l'homme est tant incapable de rendre autrui heureux que de jouir luimême du bonheur.

16 Pour parler proprement, toutefois, ces défauts du caractère ne devraient pas être attribués aux individus qui en sont affectés, mais aux effets écrasants du système dans lequel ils ont été élevés. Mais les effets de ce principe du gain que rien ne restreint sont encore plus lamentables dans les classes laborieuses, celles qui sont employées dans les parties opérationnelles des manufactures. Car la plupart de ces secteurs sont plus ou moins défavorables à la santé et au moral des adultes. Et pourtant les parents n'hésitent pas à sacrifier le bien-être de leurs enfants en les livrant à des tâches par lesquelles la constitution de leurs esprits et de leurs corps est rendue considérablement inférieure par rapport à ce qu'elle aurait pu et dû être dans un régime de prévoyance et d'humanité générales. Il y a à peine 30 ans, les parents les plus pauvres estimaient que quatorze ans était un âge suffisant pour que leurs enfants commencent à travailler régulièrement. Et leur jugement était correct, car c'est à ce moment-là de leur vie qu'ils acquéraient, à travers le jeu et l'exercice physique à l'air libre, les bases d'une constitution saine et robuste; et, s'ils n'étaient pas tous initiés à l'étude par les livres, on leur avait au moins appris la bien plus utile connaissance de la vie domestique, qui ne pouvait pas ne pas leur être familière à quatorze ans et qui leur était plus précieuse, au fur et à mesure qu'ils grandissaient et qu'ils devenaient chefs de famille, que la moitié de leurs salaires actuels, car c'était elle qui leur apprenait comment bien dépenser leurs gains.

17 Il faudrait rappeler aussi que, à cette époque-là, douze heures par jours, incluant le temps d'un repos et de repas réguliers, étaient tenues pour suffisantes pour extraire

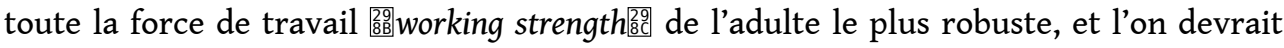
également remarquer que les jours fériés étaient bien plus fréquents qu'aujourd'hui dans les différentes régions du Royaume. A cette même époque, les classes laborieuses étaient généralement éduquées par l'exemple de quelque propriétaire de terres, ce qui 
finissait par créer un intérêt commun entre les partenaires, par lesquels moyens même le paysan le plus humble était considéré comme appartenant à une famille respectable, et comme étant en un certain sens l'un de ses membres.

Dans de telles conditions, les classes inférieures non seulement jouissaient d'un degré considérable de confort matériel, mais avaient aussi souvent la possibilité de pratiquer des exercices physiques et des amusements sains et rationnels. Ainsi, ils s'attachent fortement à ceux dont ils dépendaient, leurs services étaient fournis volontairement, et de bons procédés réciproques reliaient les partenaires par les liens les plus forts de la nature humaine, faisant qu'ils se considèrent les uns les autres comme des amis qui se trouvent dans des situations différentes - les serviteurs jouissaient souvent en effet d'un confort plus stable et d'une plus grande insouciance que les maitres.

Comparez cet état de choses à la situation des classes inférieures aujourd'hui - à la nature humaine telle qu'elle est aujourd'hui, sous le nouveau régime des manufactures.

Dans les quartiers manufacturiers, la pratique commune pour les parents consiste à envoyer travailler leurs enfants des deux sexes âgés de sept-huit ans, en hiver comme en été, à six heures du matin, parfois évidemment dans l'obscurité, le froid et la neige, pour rentrer dans les ateliers, qui sont souvent chauffés à des hautes températures et contiennent une atmosphère qui est loin d'être la plus favorable à la vie humaine, et où tous ceux qui y sont employés bien souvent continuent de travailler jusqu'à midi, quand une heure pour déjeuner est accordée, après quoi ils retournent travailler pour rester souvent jusqu'à huit heures du soir.

21 Les enfants découvrent maintenant qu'ils doivent travailler sans cesse juste pour survivre à peine, ils ne se sont pas familiarisés avec des amusements innocents, sains et rationnels; ils ne peuvent pas leur consacrer le temps requis, quand bien même ils s'étaient accoutumés à en jouir. Ils ne savent pas ce que veut dire se détendre, en dehors de la simple cessation du travail. Ils sont entourés par d'autres êtres qui se trouvent dans les mêmes circonstances qu'eux; ainsi, en passant de l'enfance à la jeunesse, ils sont progressivement initiés - les jeunes hommes en particulier, mais souvent aussi les jeunes femmes - aux plaisirs séduisants de la taverne et de l'ébriété à laquelle le dur travail quotidien, l'absence de meilleures habitudes et la vacuité générale de leur esprit tendent à les préparer.

On ne peut supposer qu'un tel système d'éducation puisse produire autre chose qu'une population faible dans ses facultés tant physiques que mentales, dotée de mœurs qui détruisent leur propre bien-être ainsi que le bien-être de ceux qui l'entourent et dont on peut prévoir qu'elles vont soumettre tous les affects sociables.

Dans de telles circonstances, l'homme ne voit tout autour de lui qu'un empressement, aussi rapide qu'une voiture tirée à quatre chevaux, pour acquérir des richesses individuelles, sans se soucier de lui, de son bien-être, de ses désirs, et même de ses douleurs, en dehors d'une dégradante charité paroissiale, ne servant qu'à recouvrir le cœur de l'homme d'une protection en acier contre ses semblables ou à éduquer le tyran et l'esclave. Il travaille aujourd'hui pour un maître, demain pour un deuxième maître, ensuite pour un troisième, un quatrième, jusqu'à ce que tous les liens entre employeurs et employés soient flétris et réduits au calcul du gain immédiat que chacun des deux peut obtenir de l'autre. L'employeur considère les employés comme des simples instruments du gain, tandis que ceux-ci acquièrent une grossière férocité de caractère qui, si des mesures législatives ne seront pas judicieusement dessinées pour prévenir 
son essor et améliorer les conditions de cette classe, finira tôt ou tard par plonger le pays dans un danger formidable et peut-être insoluble.

L'objet immédiat de ces observations est de réaliser pratiquement cette amélioration et d'éloigner ainsi le danger. La seule manière dont ces objectifs peuvent être atteints est d'obtenir une Résolution du Parlement sur les points suivants :

$1:$ limiter les heures régulières de travail dans les usines mécanisées à douze heures par jour, dont une heure et demie réservée aux repas.

2 : empêcher les enfants d'être employés dans de tels établissements jusqu'à l'âge de dix ans, ou alors qu'ils ne soient employés pendant plus de six heures par jours jusqu'à leur douzième année.

3 : faire en sorte que les enfants des deux sexes ne soient pas employés dans aucun type de manufacture - après un délai qui reste à fixer - tant qu'ils ne sont pas capables de lire et d'écrire d'une manière utile, de comprendre les quatre opérations arithmétiques et que les filles ne soient pas capables de coudre leurs propres vêtements.

Ces mesures, lorsqu'elles ne sont pas déformées par des sentiments partisans ou par des notions fausses et étriquées de son propre intérêt immédiat, mais si on les considère uniquement du point de vue du bien de la nation, s'avéreront bénéfiques pour les enfants, pour les parents, pour les employeurs, pour la nation. Toutefois, tels que nous sommes éduqués à l'heure qu'il est, maints individus ne peuvent séparer les objets généraux des considérations partisanes, tandis que d'autres ne peuvent les envisager qu'au prisme du gain monétaire immédiat. On peut donc en conclure que des individus aux profils variés se trouveront être en désaccord avec certaines de ces mesures, voire avec leur totalité. J'essayerai partant d'anticiper leurs objections et de leur répondre.

Les enfants ne peuvent pas être imaginés en train d'objecter quoi que ce soit aux plans proposés: on peut facilement leur apprendre, à travers la preuve que fournit l'expérience, à les voir comme des mesures essentiellement bénéfiques pour eux pendant l'enfance, l'adolescence, l'âge d'homme et la vieillesse.

Les parents qui ont grandi au milieu de l'ignorance et des mauvaises habitudes, et qui sont par conséquent livrés à la pauvreté, pourraient dire : « Nous ne pouvons pas nous permettre d'entretenir notre enfant jusqu'à sa douzième année, sans les envoyer travailler pour gagner un salaire; nous sommes ainsi opposés à cette mesure qui nous empêche d'envoyer nos enfants dans les manufactures avant d'avoir atteint cet âgelà ».

Si les gens les plus pauvres et les plus misérables pouvaient autrefois entretenir leurs enfants jusqu'à quatorze ans, pourquoi ne pourraient-ils pas maintenant les entretenir sans un emploi régulier jusqu'à leur douzième année? Si les parents qui négligent ce devoir n'étaient pas ignorants et éduqués par des mauvaises habitudes qui rendent leurs facultés mentales inférieures à l'instinct de mainte espèce animale, ils comprendraient que, en forçant leurs enfants à travailler dans de telles conditions à un âge trop précoce, ils livrent leurs rejetons à des circonstances qui retarderont nécessairement leur croissance, les rendront particulièrement exposés aux maladies du corps et aux dégâts de l'esprit, tout en leur soustrayant la possibilité d'acquérir cette saine et robuste constitution qu'ils auraient possédé autrement et faute de laquelle ils ne peuvent guère jouir du bonheur, mais finissent par être un fardeau pour eux-mêmes, pour leurs proches et pour leur pays. 
29 En agissant de la sorte, ces parents privent aussi leurs enfants de la possibilité d'acquérir les habitudes de la vie domestique, sans une certaine connaissance de laquelle même des salaires absolus assez élevés ne peuvent leur procurer que peu d'aisance, et les classes laborieuses ne peuvent éprouver que fort peu de bonheur domestique.

30 Des enfants si précocement employés sont empêchés d'acquérir les moindres rudiments de l'apprentissage par les livres ; et, à la place de cette connaissance utile et profitable, ils vont vraisemblablement acquérir les habitudes les plus dommageables en côtoyant continuellement des gens aussi ignorants et mal éduqués qu'eux. Et l'on peut vraiment dire que, pour chaque penny que les parents gagnent à travers le travail trop précoce de leurs rejetons, ils sacrifient non seulement des pounds à venir, mais aussi la santé, le bien-être et la conduite à venir de leurs enfants. Si ce système pernicieux n'est pas supplanté par l'introduction d'un système meilleur, le mal va vraisemblablement s'étendre et empirer à chaque nouvelle génération.

31 Je n'anticipe aucune objection de la part des employeurs à propos de l'âge fixé pour accepter les enfants dans leurs manufactures, ou concernant l'obligation d'attendre que les enfants aient acquis au préalable des bonnes habitudes - car, sur la base d'une expérience suffisante pour discerner la réalité effective, j'ai constamment trouvé plus profitable de faire accéder les enfants à un emploi quotidien constant à l'âge de dix ans que de le faire à un quelconque âge plus précoce; et j'ai constaté que ces enfants, ou ces adultes, qui ont reçu une bonne éducation, deviennent les meilleurs des serviteurs, et les plus facilement dirigés dans l'exécution de tout ce qui était juste et adéquat à accomplir. Les propriétaires d'établissements très couteux peuvent soulever des objections contre la réduction du temps de travail qui est actuellement accepté. La plus grande force de cet argument, toutefois, réside en ceci, que la rente ou l'intérêt du capital dépensé dans la création d'un établissement dépend de la quantité de son produit, et que, si ces propriétaires, au lieu de pouvoir utiliser leurs travailleurs dans leurs manufactures aussi longtemps que la nature humaine peut être soumise à des efforts continus, disons pendant quatorze ou quinze heures par jour, étaient forcés à respecter une limite de douze heures de travail, le coût du produit de la manufacture serait augmenté par la rente ou l'intérêt proportionnellement plus grands rattachés à une quantité de produit moins importante. Si, néanmoins, cette loi était en vigueur dans toute l'Angleterre, ainsi qu'en Ireland et en Ecosse, comme nous le proposons, quelle que soit la différence susceptible de survenir dans le coût des produits de ces manufactures, elle serait portée par les consommateurs, et non par les propriétaires de ces établissements. Et, du point de vue de la nation, le travail exercé douze heures par jour sera obtenu d'une manière plus économique que s'il était étendu sur une durée supérieure. Toutefois, je doute qu'une manufacture quelconque, organisée de telle sorte que ses employés travaillent douze heures par jour, ne produira pas son tissu, même du point de vue du propriétaire immédiat, presque, sinon tout à fait, aussi bon marché que les manufactures où les efforts des employés sont prolongés jusqu'à quatorze ou quinze heures. Si cela ne se produisait pas d'une manière aussi ample que prévu, néanmoins, l'amélioration de la santé, le confort, tous ces acquis très utiles à la population, et la diminution du taux de pauvreté naturellement due à ce changement dans les conduites et dans les habitudes du peuple, finiront par compenser largement, du point de vue du pays, une simple augmentation relative du coût d'une marchandise quelconque. Et peut-on imaginer que le Gouvernement britannique puisse opposer 
l'éventualité d'un profit insignifiant de quelques individus au bien-être stable de tant de millions d'êtres humains ? L'employeur ne peut subir un tort lorsqu'il est obligé à agir à l'égard de ses travailleurs de la manière dont il doit agir dans l'intérêt de la nation.

Depuis l'introduction générale de machines très couteuses, la nature humaine a été forcée bien au-delà de ses capacités normales ; il s'en suit beaucoup de misère privée et de dégâts publics.

Il s'agit en effet d'une mesure plus déplorable, du point de vue de la nation, que n'importe quelle autre décision prise depuis maints siècles. Elle a bouleversé les mœurs domestiques de la grande masse du peuple. Elle les a privés du temps d'acquérir une instruction ou de jouir d'amusements rationnels. Elle leur a volé leurs biens essentiels, et, en les menant aux habitudes de la taverne et de l'ébriété, elle a empoisonné tous les avantages de leur sociabilité.

On ferait donc des lois pour emprisonner, déporter ou condamner à mort ceux qui volent quelques centimes de nos richesses, blessent l'un de nos animaux domestiques ou même une simple brindille, et on ne devrait pas faire des lois visant à restreindre ceux dont le désir du profit ne saurait être restreint autrement, à les empêcher de priver, au nom de ce désir, des millions de nos semblables de leur santé, de leur temps consacré à l'apprentissage et à l'amélioration de soi, de leur bien-être social et de toute réjouissance rationnelle? Ce système ne peut durer longtemps - il produira de luimême un remède par les maux pratiques qu'il engendre, et ce d'une manière très dangereuse pour le bien commun, du moins si le Gouvernement ne lui impose pas la direction à suivre.

Toutefois, le public est peut-être plus intéressé à la partie du plan qui préconise l'éducation des classes inférieures sous la direction et aux frais du pays. Et il est fortement souhaitable que les avantages substantiels qui jailliront de cette mesure étaient envisagés et compris d'une manière plus générale, afin que des idées mal comprises à son égard, circulant dans les milieux les plus divers, soient entièrement dissipées.

Une connaissance générale des états passés du monde avec un minimum d'expérience de la nature humaine, à l'instar de ce qui se manifeste dans les petites sectes et dans les petits partis qui nous entourent, suffit pour rendre évident pour ceux qui n'ont pas été mal instruits depuis l'enfance qu'il est possible d'apprendre aux enfants virtuellement n'importe quels habitudes et sentiments; et que ces dernières, avec les dispositions corporelles et mentales qui existent depuis la naissance dans chaque individu, constituent, en se combinant avec les circonstances générales où chacun se trouve situé, tout le caractère de l'homme.

Il est partant évident que la nature humaine peut être améliorée et formée afin qu'elle revête le caractère qu'elle devrait posséder pour l'intérêt et le bonheur de tous, et ce uniquement en dirigeant l'attention de l'humanité vers l'adoption de mesures législatives judicieusement calculées pour donner les meilleurs habitudes, les sentiments les plus justes et les plus utiles, aux nouvelles générations - et tout particulièrement à ceux qui se trouvent vivre dans des situations qui, faute de telles mesures, les exposent à absorber les pires habitudes et les sentiments les plus inutiles et nuisibles. 
38 Je pose la question à ceux qui ont étudié la science du gouvernement à partir de ces principes éclairés qui seuls devraient influencer l'homme d'État: quelle différence, du point de vue du bien de la nation, entre, d'une part, un individu éduqué au milieu d'habitudes qui lui donnent la santé, la tempérance, l'ardeur à la tâche, des principes corrects du jugement, de la prévoyance et une bonne conduite générale, et, d'autre part, un individu éduqué dans l'ignorance, la paresse, l'intempérance, les capacités de juger diminuées, et les mœurs générales vicieuses? Le premier n'est-il pas réellement plus utile pour l'État, plus porteur de force politique, que de nombreux exemplaires ressemblant au deuxième? N'y a-t-il pas, dans les dominions britanniques, plusieurs millions d'individus sur lesquels on peut opérer cette différenciation? Et, si une transformation peut être accomplie, qui agit d'une manière si fondamentale sur le bien-être de ces individus et, au travers d'eux, sur chaque membre de l'Empire, le premier devoir du Gouvernement et de la nation ne consiste-t-il pas à mettre immédiatement en pratique les moyens qui peuvent opérer une telle transformation?

rrait-il que des mesures d'une telle importance soient escamotées, et les intérêts supérieurs de ce pays compromis, parce qu'un certain parti souhaite que ses principes particuliers soient imposés aux jeunes esprits, ou qu'un autre parti craint que les avantages de ce système légal amélioré seront assez importants pour augmenter excessivement la popularité et le pouvoir des Ministres qui l'auront introduit?

41 La fin de ces erreurs est - j'en suis sûr - très proche, et alors le Gouvernement ne sera plus forcé de sacrifier la bienfaisance et le bien-être de la grande masse du peuple et de l'Empire devant les préjugés d'un nombre relativement exigu d'individus, dont l'éducation les égare jusque dans l'évaluation de leur propre sécurité et de leur intérêt.

De toute évidence, une mesure très clairement susceptible de rendre un plus grand service à des millions de nos semblables qu'aucune autre jamais adoptée ne saurait être retardée plus longtemps parce qu'un certain parti au sein de l'État pourrait faussement supposer qu'elle affaiblirait son influence sur l'esprit public, sauf si c'était ledit parti qui dirigerait à lui tout seul un tel plan, dont la direction toutefois, c'est évident, ne saurait être laissée à un seul parti par l'intelligence de notre époque; ou encore parce que d'autres, éduqué par des principes tout à fait contraires, pourrait imaginer qu'un système national d'éducation pour les pauvres et les classes inférieures placé sous la responsabilité du Gouvernement, mais géré et dirigé dans ses détails par le pays, attribuerait un pouvoir dangereux aux Ministres de la Couronne.

De tels sentiments ne peuvent exister dans des esprits libres de considérations partisanes, qui désirent sincèrement le bien de leurs semblables, qui n'ont pas d'intérêt particulier à affirmer, qui souhaitent soutenir et renforcer le Gouvernement pour que le Gouvernement puisse être davantage capable d'adopter des mesures décisives et efficaces ayant en vue l'amélioration générale de la vie du peuple.

Ainsi, au nom des millions de pauvres et ignorants laissés de côté, dont les habitudes et les sentiments ont été formés jusqu'ici de manière à les rendre misérables, j'en appelle au Gouvernement britannique et à la Nation britannique pour qu'ils unissent leurs forces afin d'organiser un système visant à éduquer et à instruire ceux qui sont actuellement non éduqués et non instruits du point de vue de toute fin bonne et utile; et qu'ils fassent cesser, par un système de prévention clair, simple et pratique, l'ignorance, le vice et la misère qui augmentent rapidement partout dans l'Empire - car 
"éduquez un enfant à emprunter le droit chemin, et il ne l'abandonnera pas une fois devenu adulte ».

\section{Karl Marx : « Enquête ouvrière pour la Revue socialiste »}

Aucun gouvernement (monarchiste ou républicain bourgeois) n'a osé entreprendre une enquête sérieuse sur la situation de la classe ouvrière française. Mais, en revanche, que d'enquêtes sur les crises agricoles, financières, industrielles, commerciales, politiques.

Les infamies de l'exploitation capitaliste révélées par l'enquête officielle du gouvernement anglais; les conséquences légales que ces révélations ont produites (limitation de la journée légale de travail à 10 heures, lois sur le travail des femmes et des enfants, etc.) ont rendu la bourgeoisie française encore plus craintive des dangers que pourrait représenter une enquête impartiale et systématique.

En attendant que nous puissions amener le gouvernement républicain à imiter le gouvernement monarchique de l'Angleterre, à ouvrir une grande enquête sur les faits et méfaits de l'exploitation capitaliste, nous allons, avec les faibles moyens dont nous disposons, essayer d'en commencer une. Nous espérons être soutenus dans notre œuvre, par tous les ouvriers des villes et des campagnes qui comprennent qu'eux seuls peuvent décrire en toute connaissance de cause les maux qu'ils endurent; qu'eux seuls, et non des sauveurs providentiels peuvent appliquer énergiquement les remèdes aux misères que l'exploitation capitaliste leurs font subir.

1. Quel est votre métier?

2. Est-ce que l'atelier où vous travaillez appartient à un capitaliste ou à une compagnie d'actionnaires?

3. Donnez le nombre de personnes employées.

4. Donnez leur âge et leur sexe.

5. Quel est âge le plus jeune auquel les enfants (garçons et filles) sont admis?

6. Donnez le nombre de surveillants et des autres salariés qui ne sont pas des salariés ordinaires.

7. Y a-t-il des apprentis ? - Combien?

8. Outre les ouvriers ordinairement et régulièrement employés, y'en a-t-il d'autres qui ne viennent qu'à certaines saisons?

9. Est-ce que l'entreprise de votre patron travaille exclusivement ou principalement pour les chalands de la localité, pour le marché intérieur général ou pour l'exportation étrangère?

10.L'atelier est-il situé à la campagne ou à la ville?

11.Si votre atelier est situé à la campagne est-ce que votre travail industriel suffit à vous faire vivre, ou bien le combinez-vous avec un travail agricole?

12. Est-ce que votre travail est fait à la main ou avec l'aide des machines?

13. Donnez des détails sur la division du travail de votre industrie.

14. Emploie-t-on la vapeur comme force motrice?

15. 지을 Énumérez le nombre de chambres dans lesquelles les différentes branches de l'industrie sont pratiquées. - Décrivez la spécialité dans laquelle vous êtes employé ; parlez non seulement de la partie technique, mais encore de la fatigue musculaire et nerveuse qu'elle impose et de ses effets généraux sur la santé des ouvriers $\left.{ }^{\left[0_{0}^{2}\right.}\right]^{3}$. 
16. Décrivez les conditions hygiéniques de l'atelier : dimension des pièces, place assignée à chaque ouvrier ; ventilation, température, blanchissement des murs à la chaux, lieux d'aisance, propreté générale ; bruit des machines, poussières métalliques, humidité, etc.

17. Existe-t-il une surveillance municipale ou gouvernementale sur les conditions hygiéniques des ateliers?

18. Dans votre industrie, y a-t-il des émanations délétères spéciales engendrant des maladies spécifiques pour les ouvriers?

19. L'atelier est-il encombré de machines?

20. La force motrice, les appareils de transmission et les machines sont-elles protégées de manière à prévenir tout accident?

21. Enumérez les accidents arrivés durant votre expérience personnelle.

22. Si vous travaillez dans une mine, énumérez les mesures préventives prises par votre employeur pour ventiler, pour empêcher les explosions et autres accidents dangereux.

23. Si vous travaillez dans une fabrique de produit chimique, dans une usine, une manufacture d'objets métalliques ou toute autre industrie présentant des dangers spéciaux, énumérez les mesures de prévention prises par votre employeur.

24. Quels sont les moyens d'éclairage de votre atelier (gaz, pétrole, etc.) ?

25. En cas d'incendie, les moyens de fuite sont-ils suffisants?

26. En cas d'accident votre employeur est-il obligé légalement d'indemniser l'ouvrier ou sa famille?

27. Sinon, a-t-il jamais indemnisé ceux à qui il est arrivé malheur pendant qu'ils travaillaient à l'enrichir?

28. Y a-t-il un service médical dans votre atelier?

29. Si vous travaillez à votre domicile, décrivez l'état de votre chambre de travail. vous servez-vous seulement d'outil ou de petite machine ? - vous faites-vous aider par vos enfants ou d'autres personnes (adultes ou enfants, mâles ou femelles) ? - travaillezvous pour des clients particuliers ou pour un entrepreneur ? - traitez-vous directement avec lui ou par un intermédiaire?

30. Enumérez les heures de travail quotidiennes et les jours de travail pendant la semaine.

31. Enumérez les jours fériés pendant l'année.

32. Quelles sont les interruptions de la journée de travail ?

33. Les repas sont-ils pris à des intervalles déterminés ou irrégulièrement ? Sont-ils pris en dehors de l'atelier?

34. Travaille-t-on pendant les heures des repas?

35. Si on emploie la vapeur, quand la donne-t-on, quand l'arrête-t-on?

36. $\mathrm{Y}$ a-t-il du travail de nuit?

37. Enumérez les heures de travail des enfants et des jeunes personnes en dessous de 16 ans.

38. Y-a-t-il des relais d'enfants et de jeunes personnes se remplaçant mutuellement pendant les heures de travail?

39. Les lois sur le travail des enfants sont-elles mises en vigueur par le gouvernement ou la municipalité ? Les employeurs s'y soumettent-ils?

40. Existe-t-il des écoles pour les enfants et les jeunes personnes employés dans votre métier ? S'il y en a, quelles sont les heures d'école? qui les dirige ? Qu'y enseigne-t-on? 41. Quand le travail est de nuit et de jour, quel est le système de relais? 
42. Quelle est la prolongation habituelle des heures de travail pendant la période de grande activité industrielle?

43. Les machines sont-elles nettoyées par des ouvriers spécialement engagés pour ce travail ; ou le sont-elles gratuitement par les ouvriers employés aux machines pendant leur journée de travail?

44. Quels sont les règlements et les amendes pour les retards? quand la journée de travail commence-t-elle, quand recommence-t-elle après le repas?

45. Quel temps perdez-vous en vous rendant à l'atelier et en rentrant chez vous?

46. Quels sont les contrats que vous passez avec votre employeur ? Êtes-vous engagé à la journée, à la semaine, au mois, etc. ?

47. Quelles sont les conditions stipulées pour donner ou recevoir son congé ?

48. Dans le cas de bris de contrat, quand c'est l'employeur qui est en faute, quelle est sa pénalité?

49. Quand c'est l'ouvrier qui est en faute qu'elle est sa pénalité ?

50. S'il y a des apprentis, quels sont les termes de leur contrat?

51 . Votre travail est-il régulier ou irrégulier?

52. Dans votre métier travaille-t-on seulement de certaines saisons; ou est-ce que le travail, dans les temps ordinaires, est distribué plus ou moins également pendant toute l'année ? Si vous ne travaillez qu'à de certaines saisons, comment vivez-vous dans l'intervalle?

53. Etes-vous payé au temps ou à la pièce?

54. Si vous êtes payé au temps, êtes-vous payé à l'heure ou à la journée?

55. Y a-t-il des salaires extra, pour du travail extra? Quels sont-ils?

56. Si vos salaires sont payés à la pièce, comment les fixe-t-on? Si vous êtes employé dans des industries où le travail est mesuré par la quantité ou le poids, comme c'est le cas dans les mines, votre employeur ou ses commis ont-ils recours à des tricheries pour vous frauder d'une partie de vos gains?

57. Si vous êtes payé à la pièce, fait-on de la qualité de l'article un prétexte pour des déductions frauduleuses de vos salaires?

58. Que vous soyez payé à la pièce ou au temps, quand êtes-vous payé, en d'autres mots, combien long est le crédit que vous faite à votre maître avant de recevoir le prix du travail exécuté ? Etes-vous payé après une semaine, un mois, etc., ?

59. Avez-vous remarqué que le retard dans le paiement de vos salaires vous oblige à recourir fréquemment au mont de piété 3 , payant là un haut taux d'intérêt, vous dépouillant de choses dont vous avez besoin ; de faire des dettes chez les boutiquiers, devenant leur proie parce que vous êtes leur débiteur? Connaissez-vous des cas où des ouvriers ont perdu leurs salaires par la faillite ou la banqueroute de leurs patrons?

60. Les salaires sont-ils payés directement par le patron ou par des intermédiaires (marchandeurs, etc...)?

61. Si les salaires sont payés par des marchandeurs ou d'autres intermédiaires, quels sont les termes de votre contrat?

62. Quel est le taux de votre salaire en argent au jour et à la semaine?

63. Quels sont les salaires des femmes et des enfants coopérant avec vous dans le même atelier?

64. Quel a été dans votre atelier le salaire à la journée le plus élevé pendant le mois dernier?

65. Quel a été le salaire à la pièce le plus élevé pendant le mois dernier? 
66. Quel a été votre salaire pendant le même temps, et si vous avez une famille, quels sont les salaires de votre femme et de vos enfants?

67. Les salaires sont-ils payés entièrement en argent ou autrement?

68. Si c'est votre employeur qui vous loue votre domicile quelles sont les conditions? Est-ce qu'il déduit le loyer de votre salaire?

69. Quels sont les prix des objets nécessaires, tels que : a) Loyer de votre habitation ; conditions de locations ; le nombre de pièces qui la composent, des personnes qui y demeurent : réparations, assurances ; achat et entretien du mobilier, chauffage, éclairage, tabac. b) Nourriture : pain, viande, légumes, pommes de terre, etc., laitages, œufs, poissons, beurre, huile, saindoux, sucre, sel, épiceries, café, chicorée, bière, cidre, vin, etc..., tabac. c) Habillement : pour les parents et les enfants, blanchissage, soins de propreté, bains, savons, etc. d) Frais divers : ports de lettres, emprunt et dépôts au mont-de-piété, frais d'école des enfants, d'apprentissage, achat de journaux, de livres, etc., contributions à des sociétés de secours mutuels pour des grèves, des coalitions, des sociétés de résistance, etc. e) Frais, s'il y en a, occasionnés par l'exercice de votre métier. f) Impôts

70. Essayez d'établir le budget hebdomadaire et annuel de vos revenus et de ceux de votre famille, de vos dépenses hebdomadaires et annuelles!

71. Avez-vous remarqué, durant votre expérience personnelle, une plus grande hausse dans les objets nécessaires à la vie, tels que logement, nourriture, etc., que dans le salaire?

72. Énumérez les variations dans le taux des salaires qui vous sont connues.

73. Mentionnez les abaissements de salaires dans les temps de stagnation et de crise industrielle.

74. Mentionnez les hausses de salaires dans les prétendus temps de prospérité.

75. Mentionnez les interruptions dans le travail par le changement des modes et les crises particulières et générales. Racontez vos propres chômages involontaires.

76. Comparez les prix des articles que vous produisez ou des services que vous rendez avec le prix de votre travail.

77. Citez le cas que vous connaissez d'ouvriers déplacés par l'introduction des machines ou des autres perfectionnements.

78. Avec le développement des machines et la productivité du travail, l'intensité ou la durée du travail ont-elles augmenté ou diminué ?

79. Connaissez-vous aucune augmentation de salaire comme conséquence des progrès de la production?

80. Avez-vous jamais connu des ouvriers ordinaires qui aient pu se retirer à l'âge de 50 ans et vivre sur l'argent gagné dans leur qualité de salarié ?

81. Quel est, dans votre métier, le nombre d'années pendant lequel un ouvrier de santé moyenne peut continuer à travailler ?

82. Existe-t-il des sociétés de résistance 4 dans votre métier et comment sont-elles conduites ? - envoyez leurs statuts et règlements.

83. Combien de grèves se sont produites dans votre métier pendant le cours de votre expérience?

84. Combien de temps ces grèves ont-elles duré ?

85. Étaient-elles générales ou partielles?

86. 混Avaient-elles pour but une hausse des salaires ou étaient-elles faites pour résister à une réduction des salaires; ou se rapportaient-elles à la longueur ${ }_{\text {[COC }}^{20}$ de la journée de travail ou étaient-elles causées par d'autres motifs? 
87. Quels ont été leurs résultats?

88. Parlez de l'action des Prud'hommes.

89. Votre métier a-t-il soutenu des grèves d'ouvriers appartenant à d'autres corps de métiers?

90. Parlez des règlements et des pénalités établies par votre employeur pour le gouvernement de ses salariés.

91. Y a-t-il des coalitions d'employeurs pour imposer des réductions de salaires, des augmentations de travail, pour entraver des grèves et plus généralement pour imposer leur volonté?

92. Connaissez-vous des cas où le gouvernement ait abusé des forces publiques pour les mettre au service des employeurs contre leurs employés?

93. Connaissez-vous des cas où le gouvernement soit intervenu pour protéger les ouvriers contre les exactions des maîtres et leurs illégales coalitions?

94. Le gouvernement fait-il exécuter contre les maîtres les lois qui existent sur le travail ? Est-ce que les inspecteurs remplissent leur devoir?

95. Existe-t-il dans votre atelier ou votre métier des sociétés de secours mutuel pour les cas d'accidents, de maladie, de mort, d'incapacité temporaire de travail, de vieillesse, etc. Envoyez leurs statuts et règlements.

96. Est-ce que l'entrée de ces sociétés est volontaire ou compulsive ? Est-ce que les fonds sont exclusivement sous le contrôle des ouvriers?

97. Si les contributions sont compulsives et sous le contrôle des maîtres, les retiennentils sur les salaires ? Est-ce qu'elles sont rendues à l'ouvrier quand il donne congé ou est expulsé ? Connaissez-vous des cas où des ouvriers ont bénéficié de soi-disant caisses de retraite contrôlées par les patrons, mais dont le capital constituant est prélevé sur les salaires des ouvriers?

98. Y a-t-il des sociétés coopératives dans votre métier ? Comment sont-elles dirigées ? Est-ce qu'elles emploient des ouvriers du dehors de la même façon que les capitalistes le font?

99. Existe-t-il dans votre métier des ateliers où les rétributions des ouvriers sont payées en partie sous le nom de salaires et en partie sous le nom de prétendues coparticipations dans les profits ? Comparez les sommes reçues par ces ouvriers et celles reçues par d'autres ouvriers où il n'existe pas de prétendue coparticipation dans les profits. Enumérez les engagements des ouvriers vivant sous ce régime. Peuvent-ils faire des grèves etc., ou leur est-il simplement permis d'être les humbles serviteurs de leurs maîtres?

100. Quelles sont les conditions générales, physiques, intellectuelles, morales, des ouvrières et ouvriers employés dans votre métier?

101. Observations générales.

\section{NOTES}

1. [8:Le texte est celui de la deuxième édition des Observations, mis en ligne par la section anglaise des Archives Internet des Marxistes et accessible à cette adresse : https://www.marxists.org/ 
reference/subject/economics/owen/observations.htm. Il a été traduit de l'Anglais par Andrea Cavazzini. NdE 80 .

2. Suivant les données fournies par le Population Act de 1811, on constate que, en Angleterre, en Écosse et au Pays de Galles, il y a 895.998 familles travaillant principalement dans l'agriculture, 1.129.049 familles principalement employées par le commerce et la manufacture, 640.500 individus dans l'Armée et la Marine et 519,168 familles qui ne travaillent dans aucun de ces secteurs. Ce qui veut dire que le commerce emploie presque moitié plus que l'agriculture et que la population des agricultures est un tiers du total.

3. [. [9: référence présente des lacunes aux points 15 et 86 (cf. https://www.marxists.org/francais/marx/ works/1880/04/enquete.htm). D'autres éditions de ce texte circulent avec les lacunes corrigées (cf. la publication dans la revue Travailler $\mathrm{n}^{\circ} 12,2004$, qui suit l'édition de Marx dans la Pléiade par

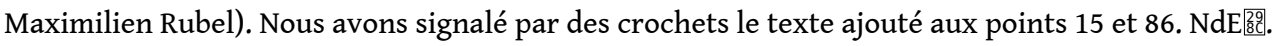

\section{RÉSUMÉS}

Documents des premières formes d'enquête militante dans le monde ouvrier par un pionnier du mouvement coopératif et par Karl Marx

\section{INDEX}

Mots-clés : Marx, classes laborieuses, manufactures, enquête, socialisme, éducation, ouvriers, classe ouvrière, capitalisme, révolution industrielle 